\title{
Catecholamine-Induced Cardiomyopathy associated with Neuroblastoma and Treated with Extracorporeal Membrane Oxygenation as a Bridge to Recovery
}

\author{
Junggu Yi, M.D. ${ }^{1}$, Si Oh Kim, M.D., Ph.D. ${ }^{1}$, Jun-mo Park, M.D., Ph.D. ${ }^{1}$, Sung-hye Byun, M.D. ${ }^{2}$, Hoon Jung, M.D. ${ }^{1}$, \\ and Seong Wook Hong, M.D., Ph.D. ${ }^{1}$ \\ 1Department of Anesthesiology and Pain Medicine, School of Medicine, Kyungpook National University, Daegu; '²epartment of Anesthesiology and Pain Medicine, \\ Catholic University of Daegu School of Medicine, Daegu, Korea
}

Catecholamine-induced cardiomyopathy associated with neuroblastoma is rarely reported. We report a case of catecholamineinduced cardiomyopathy associated with neuroblastoma in a 33-month-old female that was treated with extracorporeal membrane oxygenation (ECMO). She was tentatively diagnosed with acute myocarditis and presented with hypertension. Because of rapid patient deterioration despite pharmacological treatments, ECMO was applied. ECMO can be helpful in cases of catecholamine-induced cardiomyopathy associated with neuroblastoma.

Key Words: cardiomyopathies; catecholamines; extracorporeal membrane oxygenation; hypertension; myocarditis; neuroblastoma.

Neuroblastoma is the most common solid tumor in childhood and may present with signs and symptoms suggestive of excessive catecholamine release. One of the most fatal complications of neuroblastoma is catecholamine-induced cardiomyopathy presenting with heart failure. We present a rare case of catecholamine-induced cardiomyopathy with neuroblastoma in a female child presumptively diagnosed with acute myocarditis and treated with extracorporeal membrane oxygenation (ECMO).

\section{Case Report}

A healthy 33-month-old female child (height $110 \mathrm{~cm}$, body weight $12 \mathrm{~kg}$ ), who presented with a three-day history of fever (body temperature $38.2^{\circ} \mathrm{C}$ ) and cough, accompanied by dyspnea, was referred to our hospital for suspected acute myocarditis. Her initial blood pressure (BP) was $150 / 115 \mathrm{mmHg}$, heart rate (HR) was 170 beats per minute (bpm), and the arterial oxygen saturation as measured with pulse oximetry was $98 \%$. On physical examination, heart sounds were regular without murmur

Received on June 25, 2015 Revised on September 4, 2015 Accepted on September 22, 2015

Correspondence to: Seong Wook Hong, Department of Anesthesiology and Pain Medicine, School of Medicine, Kyungpook National University Hospital, 130 Dongdeok-ro, Jung-gu, Daegu 41944, Korea Tel: +82-53-420-5863, Fax: +82-53-426-2760 Email: hsuaa@naver.com

*No potential conflict of interest relevant to this article was reported. and her abdomen was soft and flat without any palpable mass. A plain chest radiograph showed cardiomegaly (cardiothoracic ratio $57 \%$ ) and perihilar patchy infiltrates in the right lung field; an electrocardiogram showed sinus tachycardia. Polymerase chain reaction tests were performed to identify any virus present in nasopharyngeal secretions; the results were negative. Administration of $12 \mathrm{mg}$ intravenous furosemide 


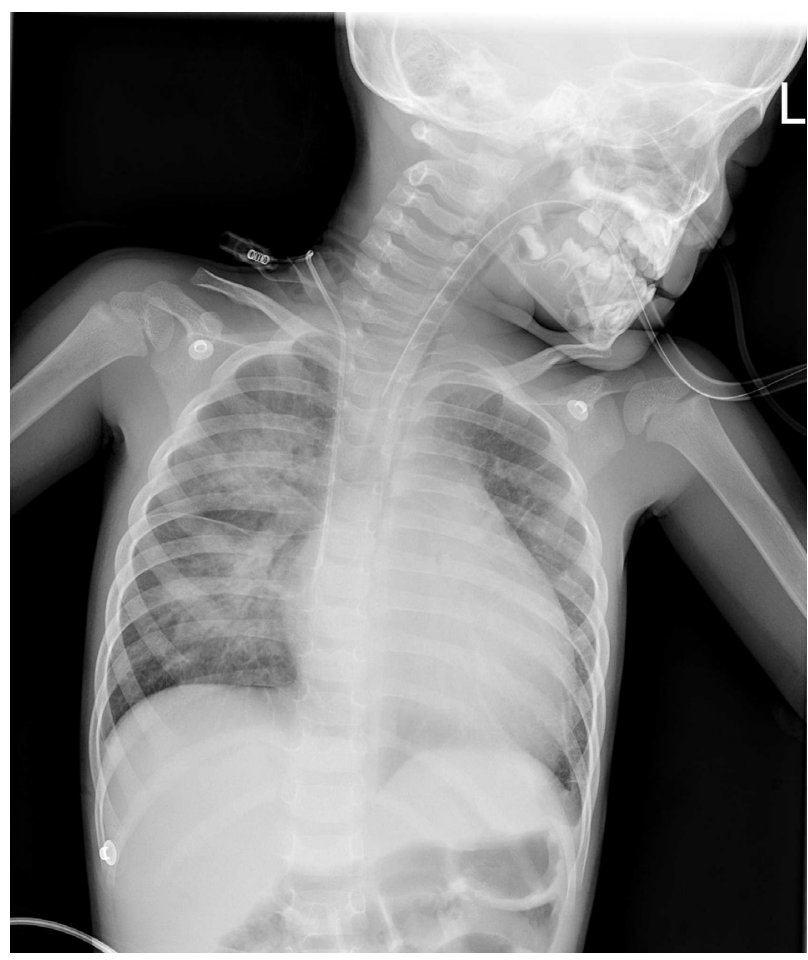

Fig. 1. Preoperative plain chest radiograph shows cardiomegaly and bilateral alveolar infiltrates.

and an infusion of dobutamine at the rate of $10 \mu \mathrm{g} / \mathrm{kg} / \mathrm{min}$ did not reduce her dyspnea. After two days of hospitalization, the patient developed excessive sweating with cyanosis. An electrocardiogram revealed sinus tachycardia with ST elevation, and a plain chest radiograph showed increased cardiomegaly (cardiothoracic ratio 59\%) and bilateral alveolar infiltrates (Fig. 1). Laboratory studies revealed abnormalities in the cardiac enzymes (creatine kinase-MB 9.4 $\mathrm{ng} / \mathrm{mL}$, troponin I $1.45 \mathrm{ng} / \mathrm{mL}$, pro-brain natriuretic peptide $>30,000 \mathrm{pg} / \mathrm{mL}$ ) and liver function test results (aspartate aminotransferase $375 \mathrm{U} / \mathrm{L}$, alanine aminotransferase $214 \mathrm{U} /$ L). Echocardiography demonstrated a markedly dilated left ventricle (left ventricle end diastolic diameter, $49.93 \mathrm{~mm}$ ), severely decreased left ventricle systolic function (ejection fraction of $12 \%$ ) and moderate mitral regurgitation. Therefore, the patient was scheduled for emergency surgery for implementation of ECMO therapy. Via median sternotomy, an 18-Fr cannula was inserted in the right atrium for drainage, after which aortic cannulation was performed on the ascending aorta. After ECMO was started, a 13-Fr vent catheter was inserted through the right upper pulmonary vein into the left ventricle to relieve the pressure. Anesthesia

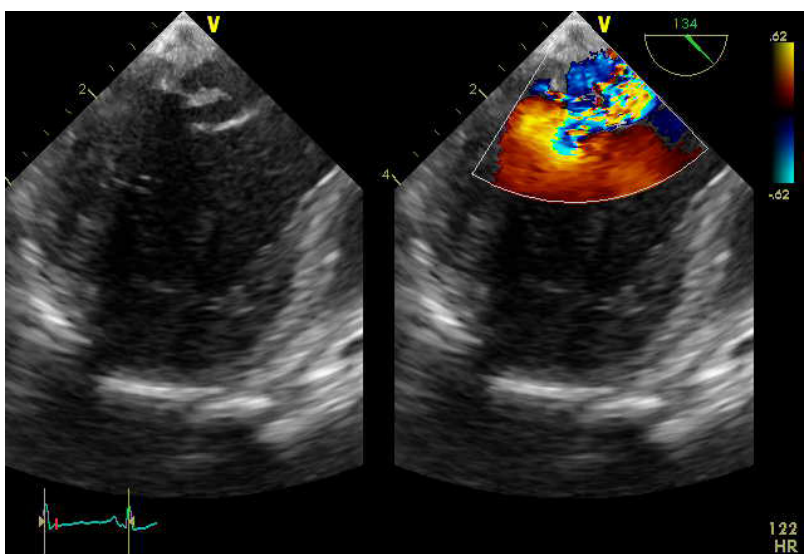

Fig. 2. Transesophageal echocardiography shows severe mitral regurgitation due to ruptured chordae tendineae. HR: heart rate.

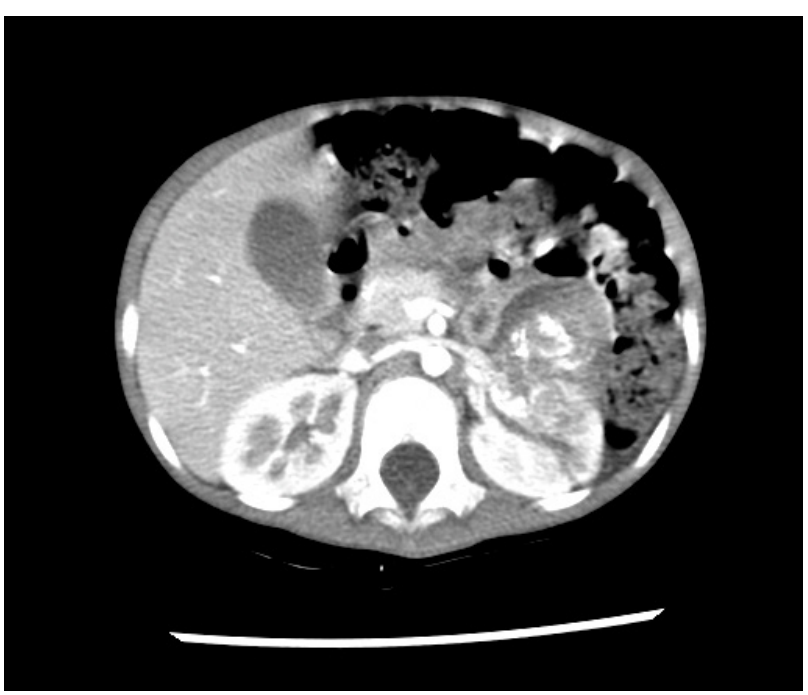

Fig. 3. Thoracic and abdominal computed tomography angiography reveals a $4-\mathrm{cm}$, heterogeneous mass in the left kidney, consistent with neuroblastoma.

was induced with $2 \mathrm{mg}$ midazolam and $10 \mathrm{mg}$ rocuronium bromide and then maintained with sevoflurane $1-2 \mathrm{vol} \%$, supplemented by fentanyl at a rate of $0.1-0.3 \mu \mathrm{g} / \mathrm{kg} / \mathrm{min}$. A bolus of fentanyl $(2 \mu \mathrm{g} / \mathrm{kg})$ was administered to bring down an elevated HR (164 bpm) after the induction of anesthesia, but the HR did not decrease easily. The BP was subsequently measured by inserting an invasive radial arterial line. Throughout the intraoperative course, the BP and HR continued to remain high, ranging from $130-100 \mathrm{mmHg} / 50-90$ $\mathrm{mmHg}$ and 130-170 bpm, respectively. On postoperative day 4, a follow-up echocardiography showed improved left ventricle wall motion (ejection fraction of $40 \%$ ). An operation was therefore performed to wean the patient from the 
Table 1. Levels of plasma and urine catecholamines and their metabolites in our patient

\begin{tabular}{lcc}
\hline & Values & Normal range \\
\hline Plasma epinephrine & $75.2 \mathrm{pg} / \mathrm{mL}$ & $18-440 \mathrm{pg} / \mathrm{mL}$ \\
Plasma norepinephrine & $\geq 2,000.0 \mathrm{pg} / \mathrm{mL}$ & $170-1,470 \mathrm{pg} / \mathrm{mL}$ \\
Plasma dopamine & $1,235.8 \mathrm{pg} / \mathrm{mL}$ & $0-20 \mathrm{pg} / \mathrm{mL}$ \\
Urine vanillylmandelic acid & $9.44 \mathrm{mg} / \mathrm{day}$ & $1.20-6.52 \mathrm{mg} / \mathrm{day}$ \\
Urine metanephrine & $0.61 \mathrm{mg} / 24 \mathrm{~h}$ & $<0.71 \mathrm{mg} / 24 \mathrm{~h}$ \\
\hline
\end{tabular}

ECMO. Mitral valvuloplasty was additionally performed to treat severe mitral regurgitation due to ruptured chordae tendineae, which had been found on intraoperative transesophageal echocardiography (Fig. 2). During the postoperative period, the patient was hypertensive at $120-200 \mathrm{mmHg} / 50$ $115 \mathrm{mmHg}$ and tachycardic at $110-150 \mathrm{bpm}$. Therefore, sodium nitroprusside was infused intravenously at a rate of 0.5 to $1.0 \mu \mathrm{g} / \mathrm{kg} / \mathrm{min}$, along with nicardipine at 1 to $2 \mu \mathrm{g} / \mathrm{kg} /$ min. Propranolol was also intermittently administered for hypertension and tachycardia during the postoperative period. However, the patient's BP was not easily controlled.

For further evaluation of perioperative hypertension, thoracic and abdominal computed tomography angiography was performed, revealing a 4-cm heterogeneous mass in the left kidney, consistent with neuroblastoma (Fig. 3). Urine and plasma catecholamine analysis also showed significant increases in the excretion of urine and plasma catecholamines and their metabolites (Table 1). The patient was transferred to another pediatric hospital for further evaluation and management, where she underwent nephrectomy and recovered without any complications.

\section{Discussion}

Catecholamine-induced cardiomyopathy associated with neuroblastoma has rarely been reported; however, it is a well-known complication caused by pheochromocytoma. Most cases have been of dilated cardiomyopathy presenting with heart failure. Some patients presented with hypertension and all showed increased urine catecholamine levels. Therefore, hypertension itself is not an essential factor of cardiomyopathy, but the result of excessive catecholamine release, which leads to cardiac muscle damage and downregulation of adrenergic receptors.[1-5] The pathogenesis of cardiomyopathy is multifactorial. Catecholamine-induced vasoconstriction or coronary vasospasm, free radical generation, chronic tachycardiomyopathy secondary to a hyperadrenergic state, -adrenergic receptor downregulation, and promotion of calcium influx into sarcolemma are the primary etiogenetic factors.[6] Cardiomyopathy in all above-mentioned cases improved after treatment of the neuroblastoma. The management of catecholamine-induced cardiomyopathy is mainly by using inotropic and antihypertensive agents, including angiotensin-converting enzyme inhibitors, angiotensin receptor blockers, and diuretics. Antihypertensive agents should be chosen with proper understanding of heart failure and the severity of outflow obstruction.[4] In our patient, because cardiomyopathy deteriorated despite pharmacological management, ECMO was implemented as a bridge to recovery, which provided time to restore heart functions without vital organ damage. Till now, there has been one case of catastrophic catecholamine-induced cardiomyopathy associated with pheochromocytoma, rescued by performing ECMO.[7]

An interesting point in our case was the finding of ruptured mitral chordae tendineae when she was weaned from ECMO. Shiraishi et al[8] reported that acute heart failure due to rupture of mitral chordate tendineae in infants is attributed to diverse causes. While the most common underlying disease is Kawasaki disease (10 cases among 95), there is no case of catecholamine-induced cardiomyopathy. It is possible that the placing of the vent catheter into the left ventricle was the reason for rupture of the mitral chordae tendineae.

Catecholamine-induced cardiomyopathy associated with neuroblastoma is rare but life-threatening, and care should be taken to ensure that it is managed with the proper pharmacological agents. Moreover, ECMO could be the treatment of choice as a bridge to recovery if the patient deteriorates rapidly despite pharmacological treatments. To that end, a pediatrician should thoroughly evaluate the etiology 
of cardiomyopathy in such patients.

\section{References}

1) Singh HR, Paules M, Forbes TJ, Zilberman MV: Reversible dilated cardiomyopathy and neuroblastoma. J Pediatr 2005; 147: 271.

2) Lee YH, Lee HD, Lee YA, Lee YS, Jung JA, Hwang $\mathrm{GG}$, et al: Ganglioneuroblastoma presenting as dilated cardiomyopathy. Arch Dis Child 2003; 88: 162-4.

3) Carlson P, Jefferies JL, Kearney D, Russell H: Refractory dilated cardiomyopathy associated with metastatic neuroblastoma. Pediatric Blood Cancer 2010; 55: 736-8.

4) Kato M, Hirata S, Kikuchi A, Ogawa K, Kishimoto H, Hanada R: Neuroblastoma presenting with dilated cardiomyopathy. Pediatr Blood Cancer 2008; 50: 391-2.

5) Kwok SY, Cheng FW, Lo AF, Leung WK, Yam MC,
Li CK: Variants of cardiomyopathy and hypertension in neuroblastoma. J Pediatr Hematol Oncol 2014; 36: e158-61.

6) Sardesai SH, Mourant AJ, Sivathandon Y, Farrow R, Gibbons Do: Phaeochromocytoma and catecholamine induced cardiomyopathy presenting as heart failure. $\mathrm{Br}$ Heart J 1990; 63: 234-7.

7) Suh IW, Lee CW, Kim YH, Hong MK, Lee JW, Kim JJ, et al: Catastrophic catecholamine-induced cardiomyopathy mimicking acute myocardial infarction, rescued by extracorporeal membrane oxygenation (ECMO) in Pheochromocytoma. J Korean Med Sci 2008; 23: 350-4.

8) Shiraishi I, Nishimura K, Sakaguchi H, Abe T, Kitano M, Kurosaki K, et al: Acute rupture of chordate tendineae of the mitral valve in infants: a nationwide survey in Japan exploring a new syndrome. Circulation 2014; 130: 1053-61. 